\title{
On the Clock Paradox
}

\author{
A. Abdesselam \\ Department of Physics, College of Science, \\ University of Tabuk, Saudi Arabia
}

doi: 10.19044/esj.2016.v12n21p130 URL:http://dx.doi.org/10.19044/esj.2016.v12n21p130

\begin{abstract}
Special Relativity presents before us several thought challenging paradoxes. The most famous one is the clock or twin paradox which arises from the well-known time dilation phenomenon. In this paper, we will give a brief but pedagogical treatment of this paradox. The crucial point is that the relativity of simultaneity, for events happening at different places, plays a fundamental role in understanding the apparent disagreement between the rest and the moving observers. Once this has been taken care of, the disagreement and the paradox would disappear.
\end{abstract}

Keywords: Clock or twin paradox, inertial frame, simultaneity, synchronisation

\section{Principles of Relativity}

The Special Theory of Relativity is based on two postulates ${ }^{1}$, namely: the relativity of physics laws and the constancy ${ }^{2}$ of the speed of light (Einstein, 1905). The second postulate is particularly handy in deriving the theory results ${ }^{3}$. Therefore, most, if not all textbooks on special relativity, use light signals and mirrors to derive the well-known formulas of time dilation and Length contraction (Kogut, 2001):

$$
\begin{aligned}
& T=\gamma T^{\prime} \\
& L=L^{\prime} / \gamma
\end{aligned}
$$

where $T$ and $L$ are time and space intervals as measured in the rest inertial frame. Also, $T^{\prime}$ and $L^{\prime}$ are the corresponding measurements in the moving frame. $\gamma$ is the usual factor $1 / \sqrt{1-v^{2} / c^{2}}$.

${ }^{1}$ It appears, however, that one could also obtain the results of the special theory from the relativity principle alone (see references in (Lee \& Kalotas, 1975; Levy-Leblond, 1976)).

2 This means that the speed of light in vacuum has the same value in all directions and in all inertial reference frames. We leave here the question of the conventionality of any one-way speed aside (see reference (Capria, 2001)).

${ }^{3}$ The isotropy of space is also implicitly assumed. 
There is another very important result of special relativity which is the relativity of simultaneity of events happening at different locations. Based on the fact that it turns out that the relativity of simultaneity is the key to understanding most of the paradoxes appearing in special relativity, we gave a very brief review of it in the next section. At the same time, we also touch on the clocks synchronisation problem.

\section{Relativity of Simultaneity}

Two similar clocks are said to be synchronized if they indicate the same time all the time. Therefore, such clocks, running at the same rate, must have started working simultaneously. We will see here that this is a relative notion indeed. The question is how to start two distant clocks at the same time. Let us assume that the clocks in question are at rest relative to the moving inertial frame $S^{\prime}$ and are separated by a distance $l^{\prime}$ (see Figure 1). A light signal is emitted from the middle point $M$. Thus, each clock begins ticking once reached by this signal whose speed in all directions is $c$. Through this way, the two clocks are set to work simultaneously with respect to the observers of $S^{\prime}$.

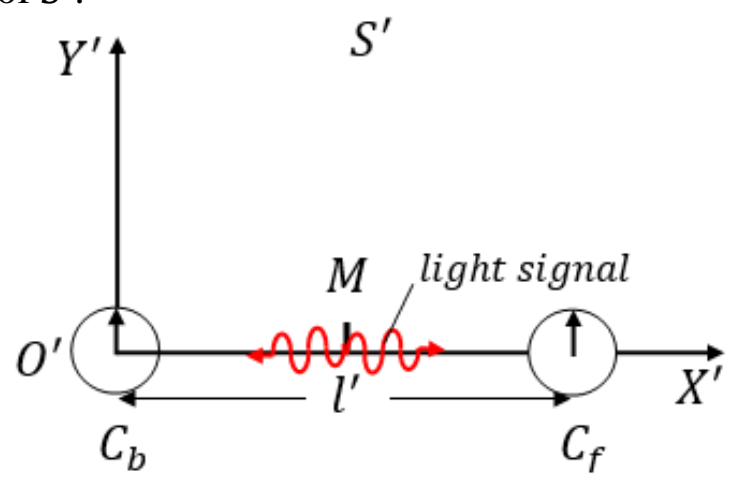

Figure 1. Synchronization of two clocks at rest in $S^{\prime}$

Let us examine this synchronisation procedure from the point of view of the observers of the frame $S$ in which the two clocks are moving along the $x$-axis with speed $v$. This is illustrated in the figure below:
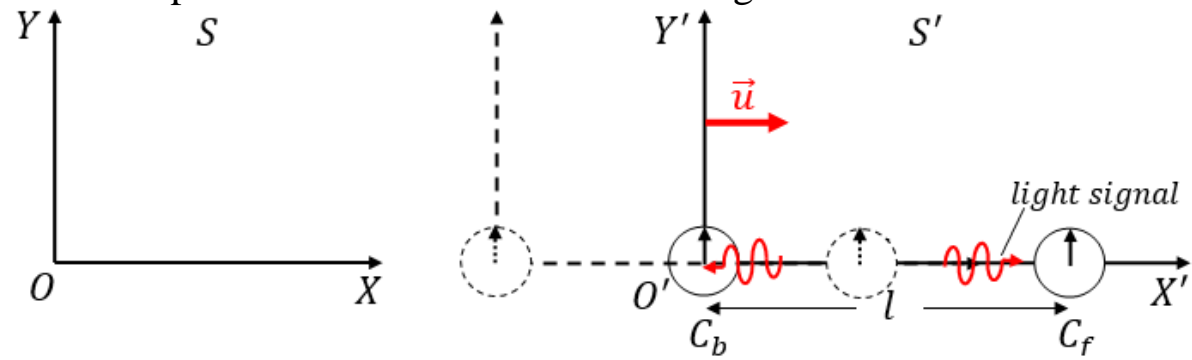

Figure 2. The $S^{\prime}$ clocks synchronization procedure as observed in $S$ 
According to these observers, the light signal reaches the back clock $C_{b}$ before it reaches the front clock $C_{f}$ (see Figure 2). This is so because $C_{f}$ is moving away from the light signal, while $C_{b}$ is moving towards it. Equally important, the speed of light is $c$ in both directions ${ }^{4}$. Thus, according to the observers of the frame $S$, the clock $C_{b}$ will start before $C_{f}$. As such, the two clocks do not start working simultaneously and are therefore not synchronized. We see that what is simultaneously relative to one frame is not necessarily the case according to another one. Therefore, the notion of simultaneity is not absolute.

We can go a step further and calculate the amount of desynchronization according to the frame $S$. Let $t_{f}$ and $t_{b}$ be the times required by light signals to reach the forward and backward clocks, respectively. According to the rest observers, the back clock starts ticking before the front clock, i.e. $t_{f}>t_{b}$. Consequently, the back clock is ahead of the front clock by an interval of time $\Delta t$. Thus, this is calculated as follows:

Or,

$$
\begin{aligned}
\Delta t & =t_{f}-t_{b} \\
& =\frac{l}{2(c-v)}-\frac{l}{2(c+v)} \\
& =\gamma^{2} \frac{v}{c^{2}} l
\end{aligned}
$$

$$
\Delta t=\gamma^{2} \frac{v}{c^{2}} l
$$

Here, all quantities are measured in the rest frame ${ }^{5}$. Notice that there may be an issue of simultaneity only for events happening at different locations. We can write the content of the previous formula in term of quantities referring to the moving frame using the time dilation and length contraction expressions given by equation (1). Then, we obtain:

$$
\Delta t^{\prime}=\frac{v}{c^{2}} l^{\prime}
$$

Furthermore, we should stress that this equation holds true only from the perspective of the frame $S$. This is because we have assumed that the clocks are in fact synchronized in $S^{\prime}$. We may as well add that the rest observers would genuinely conclude that the moving observers are using

\footnotetext{
${ }^{4}$ This is not true in Galileo relativity with ballistic light emission, where the speed of the signal in the forward direction is greater than its speed in the backward direction. The two moving clocks will still start working simultaneously and are therefore synchronized in this theory.

${ }^{5}$ It worth mentioning that the Lorentz Transformation could easily be deduced from equations (1-3). On the opposite side, given the Lorentz Transformation, these relations follow immediately (see for example Bohm, 2006).
} 
unsynchronized clocks. Consequently, they must correct their measurement in line with the previous equations. They should in fact add the lost time due to the desynchronization (the back clock being ahead of the front clock). More on this is described in the next section.

\section{Clock or Twin Paradox}

The source of this paradox ${ }^{6}$ is the time dilation formula as shown in Eq. (1). The observers at rest discovered that the moving observers' clocks are running at a slower rate when compared to their own clocks ${ }^{7}$. By symmetry, the moving observers would arrive at the same conclusion. They rightly think that they are at rest and that it is the other observers who are moving. Therefore, their clocks must be running at a slow pace. Who is right and who is wrong? This seemingly paradox has been extensively discussed in the literature (Sartori. L, 1996; Carl \& Stephen, 2001; Romer, 1959; Builder, 1957). However, let us inspect the situation more closely.

\section{Comparing One Moving Clock with the Two Rest Clocks}

Let us first examine how the rest observers do their measurement. The crucial point is that they have to compare one and only one of the moving clocks, say $C^{\prime}$, with two of their stationary synchronized clocks, say $C_{1}$ and $C_{2}$ (see Figure 3 ).

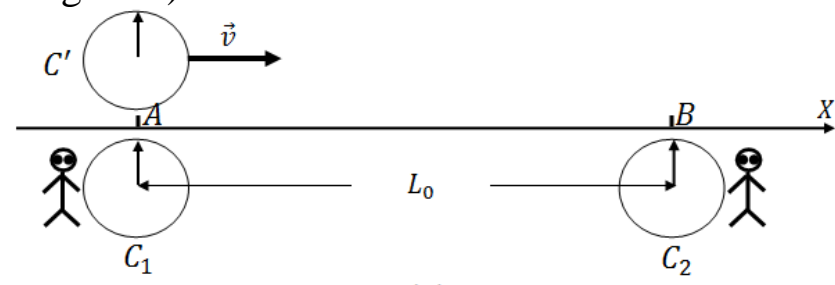

(a)

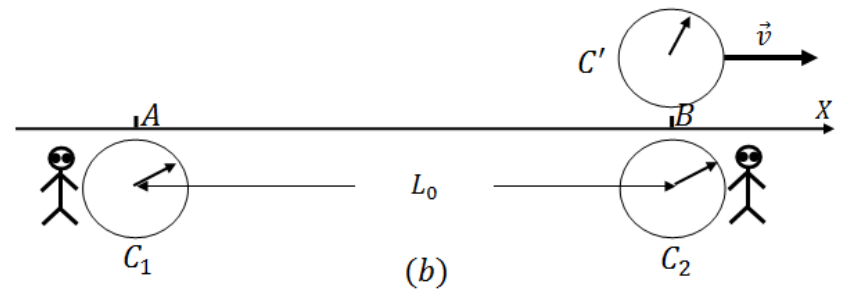

Figure 3. Comparing one moving clock with the two rest clocks at locations $A$ and $B$

\footnotetext{
${ }^{6}$ The twin paradox goes like this: A twin called $A$ travels in a round trip with a speed $v$ close to that of light. When he comes back, he finds that because of the time dilation, twin $B$ has aged more than he did. The paradox arises when we think of the problem as being symmetric: For the moving twin $A$, it is the rest twin $B$ who is travelling in the opposite direction with speed $v$. Consequently, it ought to age less, i.e. the paradox.

${ }^{7}$ This paradox concerns, of course, all types of time intervals, not only the interval between a clock's ticks.
} 
We can assume without loss of generality that $C^{\prime}$ is initially synchronized with $C_{1}$ (Figure 3-a). In other words, the rest observer standing near $C_{1}$, observes that both $C^{\prime}$ and $C_{1}$ indicate the same time, say zero. Later, when the moving clock is compared with the second clock $C_{2}$, the rest observer standing there discovers that it is lagging behind $C_{2}$ (Figure 3-b). Thus, the relation between the two readings is given by the time dilation formula:

$$
T=\gamma T^{\prime}
$$

where $T$ is the reading of $C_{2}$ and $T^{\prime}$ that of $C^{\prime}$.

By following the same procedure, the moving observers would also arrive at a similar result:

$$
T^{\prime}=\gamma T
$$

But, the moving observers, when watching those at rest doing their measurement, must raise the objection that these observers have in fact used two unsynchronised clocks, $C_{1}$ and $C_{2}$. Indeed, according to them ${ }^{8}$, clock $C_{2}$ is ahead of $C_{1}$ by an interval given by equation (2). ${ }^{9}$

$$
\Delta t^{\prime}=\gamma^{2} \frac{v}{c^{2}} l^{\prime}
$$

where $l^{\prime}$ is the distance between the two clocks as measured by the moving observer. Using $l^{\prime}=v T^{\prime}$, the above equation can be written as:

$$
\Delta t^{\prime}=\gamma^{2} \frac{v^{2}}{c^{2}} T^{\prime}
$$

The moving observers then argue that those at rest should correct their result of Eq. (4). This is done by adding an amount of time $\Delta t$ related to their own $\Delta t^{\prime}$ precisely by Eq. (5)

$$
\Delta t=\frac{\Delta t^{\prime}}{\gamma}=\gamma \frac{v^{2}}{c^{2}} T^{\prime}
$$

In other words, the net result of the rest observers should have been $T+\gamma \frac{v^{2}}{c^{2}} T^{\prime}$. Additionally, the comparison should be given as:

$$
T+\gamma \frac{v^{2}}{c^{2}} T^{\prime}=\gamma T^{\prime}
$$

By rearranging, we get:

$$
T=\gamma T^{\prime}-\gamma \frac{v^{2}}{c^{2}} T^{\prime}=\gamma T^{\prime}\left(1-\frac{v^{2}}{c^{2}}\right)=\frac{T^{\prime}}{\gamma}
$$

\footnotetext{
${ }^{8}$ According to the moving observers, the two clock $C_{1}$ and $C_{2}$ are moving in the opposite direction with speed $v$. Equation (6) is exactly equation (2) of section 3, but with the role of observers reversed.

${ }^{9}$ We could of course use equation (3) instead, which must be combined with the length contraction formula.
} 
Or,

$$
T=\frac{T^{\prime}}{\gamma}
$$

This is precisely the result obtained by the moving observers, see Eq. (5). Thus, the moving observers conclude that the reason why the rest observers get a different result is that they are using unsynchronized clocks.

Again, by symmetry, a similar reasoning using equations (2) or (3) would lead the rest observers into the same conclusion.

Therefore, because the two sets of observers will never agree on whose clocks were synchronised and whose were not, they cannot compare their measurements through this way. The way out of this puzzle consists of comparing one and only one rest clock with one and only one moving clock. No issue of simultaneity or synchronization would arise then. For this purpose, the moving clock must make a round trip. Thus, we will discuss this point in the next section.

\section{Comparing One Rest Clock with One Moving Clock}

Let us assume that the moving clock travels along the $x$-axis in the following way (see Figure 4):

- $\quad$ From the same position as the clock at rest, the moving clock accelerates towards positive $x$ in a very brief time $^{10}$ to reach a speed $v$ comparable with that of light. We assume that the two clocks were initially synchronized.

- $\quad$ The moving clock continues to move with that constant speed for a certain time. We can make this time long enough to ignore the acceleration time.

- $\quad$ The moving clock decelerates abruptly, stops, and then accelerates in the opposite direction until it reaches the speed $v$ again.

- $\quad$ The moving clock decelerates in a negligible time to stop beside the rest clock.

In order to calculate easily the time passed on each clock, let us also assume that at each unit of proper time, each clock sends a light signal to the other one (Bohm, 2006). Furthermore, we also represent the motion of the clocks and their signals in a spacetime of two dimensions (see Figure 4 and 5).

10 This assumption allows us to ignore the parts of the trip where the moving clock accelerates. A. Logunov, (Lugonov, 2005, page 165), gives a general treatment including acceleration parts and without using General Relativity. 


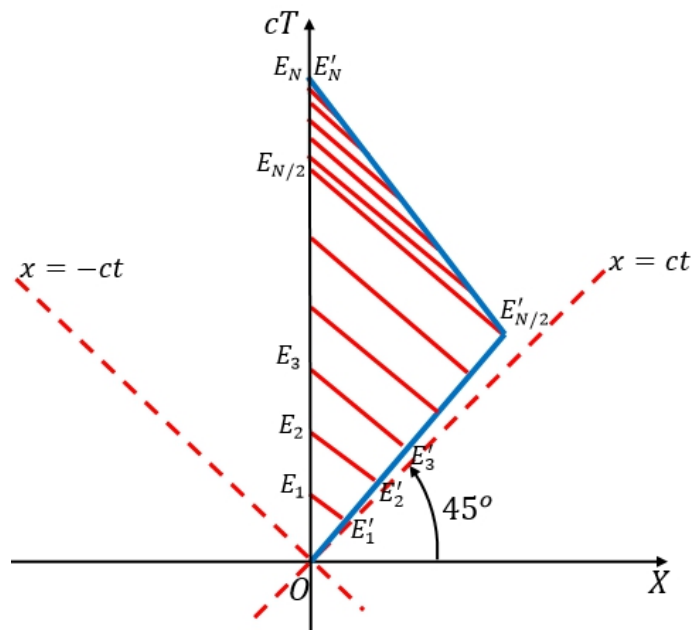

Figure 4. The world lines of the moving clock and its signals as seen in the rest frame. The signal are emitted at events $E_{i}^{\prime}$ and received at events $E_{i}$. These lines are parallel to the light world line $x=-c t$

We anticipate the result of this section. In this case, we state that both set of observers agree that, indeed, the moving clock measures a smaller time than the rest clock ${ }^{11}$.

Let us also recall the results of the well-known relativistic Doppler

Effect. A receding source emitting signals each interval $T_{0}^{\prime}$ of time as measured in its rest frame, appears to the rest observers as if it sends signals at a longer interval of time $T_{0}^{r e c}$. Therefore, this is given by:

$$
T_{0}^{r e c}=T_{0}^{\prime} \sqrt{\frac{1+\frac{v}{c}}{1-\frac{v}{c}}}
$$

Similarly, when the source is approaching, one should have:

$$
T_{0}^{a p p}=T_{0}^{\prime} \sqrt{\frac{1-\frac{v}{c}}{1+\frac{v}{c}}}
$$

Notice that the difference between the last two formulas is a reversal of the sign just before the source speed. These formulas were also obtained in the context of k-calculus developed by Bondi (Bondi, 1980; Bohm, 2006).

Now, because the moving clock moves away and towards the rest clock with the same speed $v$ for equal periods, it emits the same number of pulses $N / 2$ in each leg of the trip ( $N$ is the total number of pulses sent by the moving clock, see Figure 4). In other words, the number of signals emitted

${ }^{11}$ Time is a path dependent quantity as H. Bondi put it in reference (Bondi, 1980). 
during recession is equal to that emitted during the approach. Therefore, the rest clock should indicate a time for the whole trip which is given by:

$$
T_{\text {trip }}=\frac{N}{2} T_{0}^{r e c}+\frac{N}{2} T_{0}^{a p p}
$$

Using equations (9) and (10), we find:

$$
T_{\text {trip }}=\gamma N T_{0}^{\prime}
$$

The rest clock, then, measures a time greater than the one measured by the moving observer, i.e. $N T_{0}^{\prime}$, by the usual factor $\gamma$. Notice that we could have arrived at this result just by remembering the time dilation formula. We must have the right to do so because the rest observer is always at rest in the same inertial frame.

Let us now calculate the time of the trip as measured by the moving clock. We see from Figure 5 that the number of signals $N_{1}$ received during recession, i.e. during the outbound leg, is smaller than the number of signals $\mathrm{N}_{2}$ received during the approach, i.e. during the inbound leg of the trip. We still have of course $N_{1}+N_{2}=N$, which is the total number of signals.

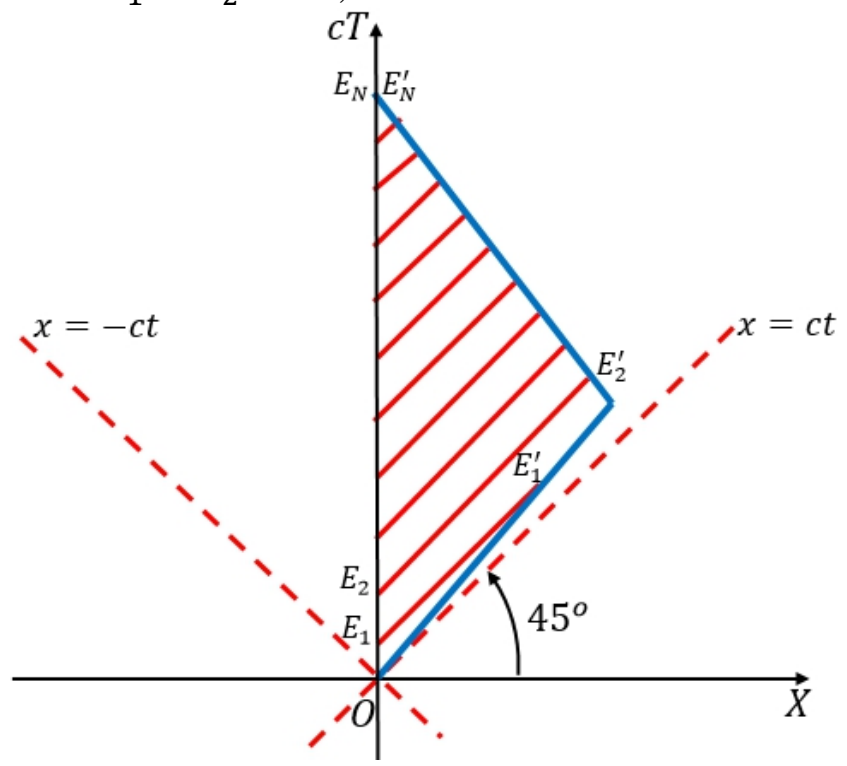

Figure 5. World lines of the signals emitted by the rest clock and received by the moving one. These lines are parallel to the light world line $x=c t$

Therefore, the moving clock records the following time:

$$
\begin{aligned}
T_{\text {trip }}^{\prime} & =N_{1} T_{0}^{\prime \text { rec }}+N_{2} T_{0}^{\prime \text { app }} \\
& =N_{1} T_{0} \sqrt{\frac{1+\frac{v}{c}}{1-\frac{v}{c}}}+N_{2} T_{0} \sqrt{\frac{1-\frac{v}{c}}{1+\frac{v}{c}}}
\end{aligned}
$$




$$
=\frac{N T_{0}}{\sqrt{1-\frac{v^{2}}{c^{2}}}}\left[1-\frac{v}{c}\left(\frac{N_{2}-N_{1}}{N}\right)\right]
$$

where $N=N_{1}+N_{2}$. Also, we show in the appendix that: ${ }^{12}$

$$
\frac{N_{2}-N_{1}}{N}=\frac{v}{c}
$$

Putting this result in the previous calculation, we get:

Or simply,

$$
T_{\text {trip }}^{\prime}=\frac{N T_{0}}{\sqrt{1-\frac{v^{2}}{c^{2}}}}\left[1-\frac{v^{2}}{c^{2}}\right]
$$

$$
T_{\text {trip }}^{\prime}=\frac{N T_{0}}{\gamma}
$$

Therefore, the moving observers agree with the rest ones. The moving clock does indeed measure a time smaller than the ones measured by the rest clock, i.e. $N T_{0}$, with exactly the expected factor.

It is important to realize that, because the moving clock accelerates in the turnaround of its journey, the situation is not symmetric anymore between the two clocks as was the case in the previous section.

Furthermore, we discuss the reason travelling observers do measure a smaller time than the rest ones ${ }^{13}$. From the point of view of the observers at rest in $S^{\prime}$, that is the moving observers, it is the observers at rest in $S$ who are moving with speed $v$ in the opposite direction. The later observers' clock should therefore run slowly according to the usual time dilation formula, $T^{\prime}=\gamma T$. If only time dilation were involved in the clock paradox, the travelling observers would expect that the rest clocks record a smaller time than what their own clocks indicate. This must be true, of course, for both the outbound and inbound legs. They would expect therefore that when they meet up with the rest observers at the end of the journey, the total times of the trip indicated by their respective clocks should be related as follow:

$$
T_{\text {trip }}=T_{\text {trip }}^{\prime} / \gamma
$$

However, this is in contradiction with equation (13) obtained through a Doppler analysis. How can we reconcile between the arguments going along with these two contradictory results? Here, once again, we have recourse to the relativity of simultaneity. The time dilation phenomenon is not the whole story. The point is that when the travelling observers do the

\footnotetext{
${ }^{12}$ I did not succeed to find this formula in the literature available to me.

${ }^{13}$ For a concrete example with numbers, see L. Sartori, chapter 6. Here we give a general treatment.
} 
turnaround at point $P$ (See Figure 6), they jump from an inertial frame to another. They jump from the inertial frame $S^{\prime}$, which is moving away from the rest observers, to another inertial frame $S^{\prime \prime}$ moving towards the rest observers. This has an important effect such that the line of simultaneous events changes. Just before the turnaround, the line of simultaneous events in the frame $S^{\prime}$ is $M_{1} P$. This line is parallel to the $x^{\prime}$-axis which makes an angle $\theta$ with the $x$-axis. This is also the same angle between the moving clock world line $O P$, which represents the $t^{\prime}$-axis in $S^{\prime}$, and the $t$-axis. This is so because the light signal world line, $x=c t$ or $x^{\prime}=c t^{\prime}$, is always a bisector in all the inertial frames ${ }^{14}$. Then, just after the turnaround, the line of simultaneous events, in $S^{\prime \prime}$, becomes $M_{2} P$. Consequently, the moving observers take account only for the time passed between $O$ and $M_{1}$ in the outbound leg and between $M_{2}$ and $G$ in the inbound leg. The time $T_{M}$ passing between the events $M_{1}$ and $M_{2}$ in the rest frame is unaccounted for by the travelling observers.

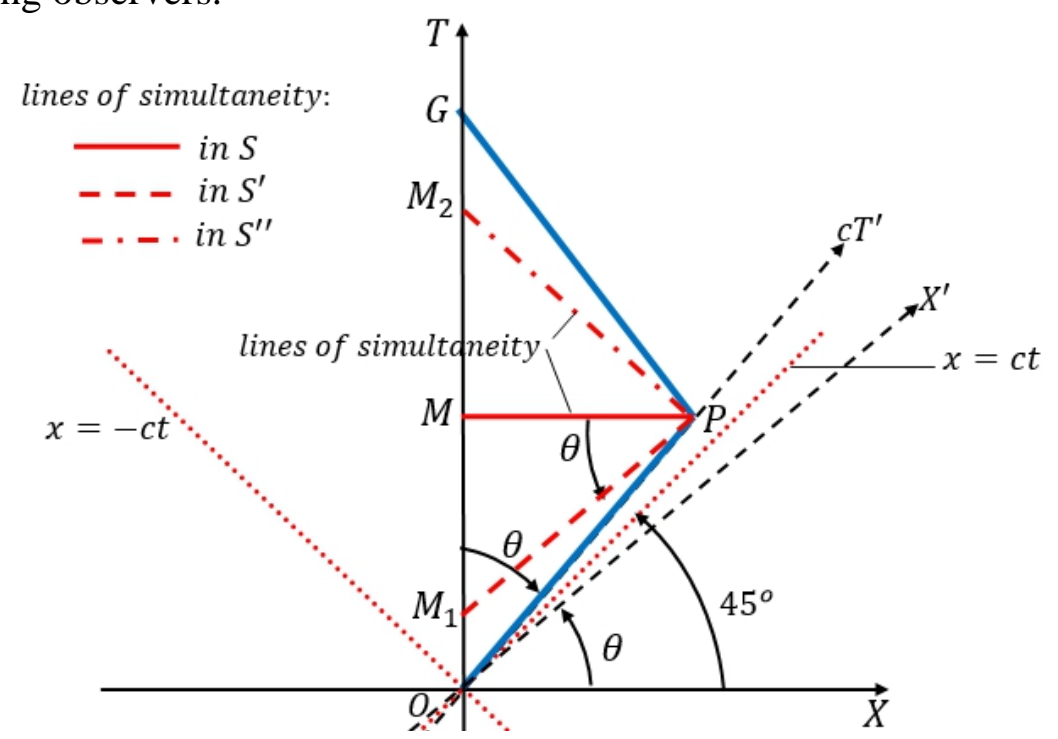

Figure 6 . Change of simultaneity line as the turnaround point

In order to correct this miscount, we added the time $T_{M}$ to the result obtained in equation (14).

$$
T_{\text {trip }}=T_{\text {trip }}^{\prime} / \gamma+T_{M}
$$

We will show in the appendix that:

$$
T_{M}=\frac{v^{2}}{c^{2}} T_{t r i p}
$$

\footnotetext{
${ }^{14}$ Notice that the events simultaneous with the event $O$ in the rest frame $S$ is the $x$-axis itself. The Line $M P$ represents the events simultaneous with $M$ in this same frame.
} 
Equation (15) then becomes:

Or,

$$
T_{\text {trip }}=T_{\text {trip }}^{\prime} / \gamma+\frac{v^{2}}{c^{2}} T_{\text {trip }}
$$

$$
T_{\text {trip }}^{\prime}=T_{\text {trip }} / \gamma
$$

which does now agree with equation (13).

\section{Conclusion}

In this paper, a short description of the twin or clock paradox was given. We showed that the clock travelling in a round trip runs effectively slower than the rest clock. The twin accompanying these clocks agrees on their measurements and no paradox arises. On the other hand, there was no attempt to explain how and why the moving clock does run slow. Perhaps, the best explanation is that we truly live in a spacetime of four dimensions. Therefore, the proper time is route or path dependent (Petkov, 2009).

\section{Acknowledgment}

I would like to thank my colleague, Dr. M. Sadiq, for reviewing the first draft of this paper and suggesting some improvements in the text. I would also like to thank the anonymous referee for several constructive comments.

\section{Appendix}

We would now like to prove equation (12). It is clear from Figure 7 that $N_{1}$ is proportional to the area $A_{1}$ (area under the triangle $O E P$ ) ${ }^{15}$ Also, $N_{2}$ is proportional to $A_{2}$ (area under the triangle $E G P$ ). Therefore,

$$
\frac{N_{2}-N_{1}}{N_{2}+N_{1}}=\frac{A_{2}-A_{1}}{A_{2}+A_{1}}
$$

15 The line $E P$, being a light signal world line, is parallel to the bisector $x=c t$. It corresponds to the signal received by the moving clock just before changing the direction of its motion. 


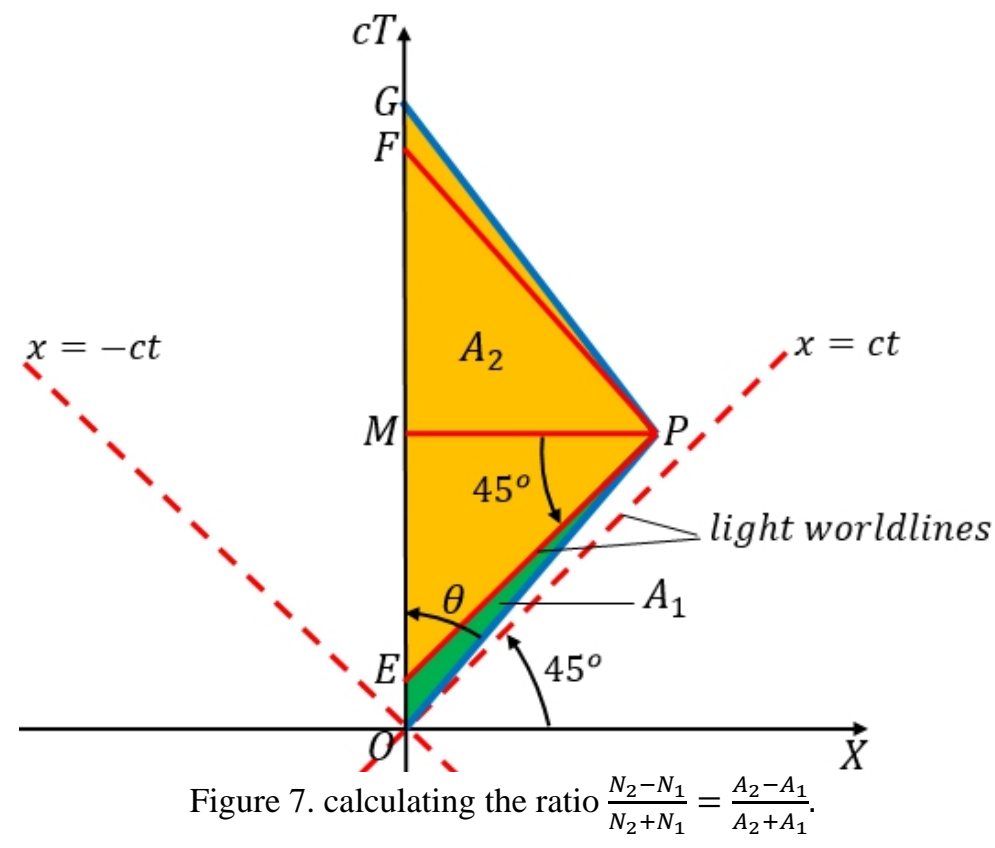

Notice also that $A_{2}+A_{1}$ is the area under the isosceles triangle $O G P$. Therefore, it is given by:

$$
A_{2}+A_{1}=\frac{O G \times M P}{2}
$$

Similarly, $A_{2}-A_{1}$ is the area under the isosceles triangle EFP. This is because $A_{1}$ is also equal to the area under the triangle $F G P$. Therefore, it is given by:

$$
A_{2}-A_{1}=\frac{E F \times M P}{2}
$$

Thus, by using the two previous equations, we have:

$$
\frac{A_{2}-A_{1}}{A_{2}+A_{1}}=\frac{E F}{O G}
$$

On another side,

Or,

$$
\tan \left(45^{\circ}\right)=\frac{E M}{M P}=\frac{E F}{2 M P}=1
$$

$$
E F=2 M P
$$

Combining equations (19) and (20), we obtain:

$$
\frac{N_{2}-N_{1}}{N_{2}+N_{1}}=\frac{2 M P}{O G}
$$

We also have, 


$$
\tan (\theta)=\frac{M P}{O M}=\frac{2 M P}{O G}=\frac{N_{2}-N_{1}}{N_{2}+N_{1}}
$$

where we used Eq. (21). But $\tan (\theta)=\frac{v}{c}$ (This is the slope of the moving clock world line. We have $x=v t=\frac{v}{c} c t$. In addition, we notice that we plotted $c t$ on the time axis and not just $t$ ). Therefore,

$$
\frac{N_{2}-N_{1}}{N_{2}+N_{1}}=\frac{v}{c}
$$

which finalizes the proof of Eq. (12).

Furthermore, we prove equation (16). We see from Figure 6 that $T_{M}=$ $M_{1} M_{2} / c$ and that

$$
M_{1} M=M P \tan (\theta)
$$

with $\tan (\theta)=v / c$ as it was just above. $M P$ represents half the distance as measured in the rest frame, which is crossed by the travelling clock. Thus, ignoring the acceleration periods, we have $M P=\frac{1}{2} v T_{\text {trip }}=\frac{1}{2} \frac{v}{c} c T_{\text {trip }}$,

where $T_{\text {trip }}$ is the duration of the whole journey as timed in the rest frame. Subsequently, we obtain the following system of equations:

From the above, we deduce that:

$$
\left\{\begin{array}{l}
M_{1} M=\frac{1}{2} M_{1} M_{2}=\frac{1}{2} c T_{M} \\
M_{1} M=M P \tan (\theta)=\frac{1}{2}\left(\frac{v}{c}\right)^{2} c T_{\text {trip }}
\end{array}\right.
$$

$$
T_{M}=\frac{v^{2}}{c^{2}} T_{t r i p}
$$

which is regarded as equation (16).

\section{References:}

Bohm D. (2006). The Special Theory of Relativity, Routledge Classics.

Bondi H. (1980). Relativity and Common Sense, Dover publications.

Capria M. M. (2001). On the Conventionality of Simultaneity in Special Relativity, Foundations of Physics, vol. 5, pp. 775-818.

Carl D. \& Stephen G. (2001). On Radar Time and the Twin Paradox, Am. J. Phys. 69, 12.

Einstein, A. (1905). Zur Elektrodynamik bewegter Körper (On the Electrodynamics of Moving Bodies), Annalen der Physik 17.

Kogut J. B. (2001). Introduction to Relativity, Academic Press.

Lee A. R. \& Kalotas T. M. (1976). Am. J. Phys. 43, 5. Levy-Leblond J., Am. J. Phys. Vol 44, 3.

Lugonov A. (2005). Henri Poincaré and relativity theory, Nauka.

Petkov V. (2009). Relativity and the Nature of Spacetime, $2^{\text {nd }}$ Ed. Springer- 
Verlag.

Romer R. H. (1959). Twin Paradox in Special Relativity, Am. J. Phys. 27, 131 (1959). Builder G., The Resolution of the Clock Paradox, Aust. J. Phys. 10, 246-262.

Sartori. L(1996). Understanding relativity: a simplified approach to Einstein'stheories, UCP. 